\title{
Probing Ionic Diffusion Dynamics through the Modulation of Polymer Shell Using Surface-Enhanced Raman Spectroscopy
}

\section{Song $X^{1}$, Phua $Z^{2 *}$, Xu $\mathbf{W}^{2}$ and Liu $^{2}$}

${ }^{1}$ Department of Materials Science and Engineering, University of Illinois at Urbana-Champaign, USA

${ }^{2}$ Department of Chemistry and Biological Chemistry, Nanyang Technological University, Singapore

\begin{abstract}
Gold nanoparticle (AuNP) encapsulated with a di-block copolymer, polystyrene-block-poly(acrylic acid) (PSPAA), is a type of core-shell nanoparticle (AuNP@PSPAA). We demonstrate the use of surface-enhanced Raman spectroscopy (SERS) to investigate the ionic diffusion of cyanide across the polymer shell. We modulate the PS blocks by using THF, incubation in water, aniline and its oligomers and lastly cholesterol, so as to increase the porosity of the polymer shell and promote the diffusion of cyanide to etch the AuNPs. Our results show that an increase in porosity will promote the etching rate and can be monitored in situ by observing the decrease in SERS intensities as the surface-active Raman molecules detaches from the AuNPs surface. Ionic diffusion of the cyanide ions is most prominent when THF is used as an organic solvent to swell the polymer shell. This new method provides us with a platform to probe ionic diffusion across hydrophobic membranes.
\end{abstract}

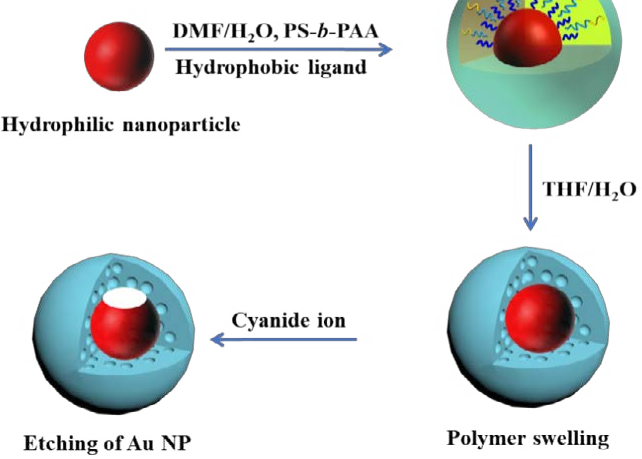

Keywords: Polymer; Nanoparticle; Swelling; Porous; Etching; Diffusion

\section{Introduction}

The diffusion of ions across a membrane can be observed in many systems such as polymer electrolyte for fuel cells [1], ion-exchange [2] and also biologically, in the cells [3]. A complete understanding of ionic diffusion, in these systems, is of fundamental importance to uncover the mechanistic details of how a membrane can function in relation to its permeability. Many studies use various polymers [4] to understand this phenomenon, by exploring its porosity [5]. More recently nanostructures have been fabricated to help us explore ionic diffusion $[6,7]$.

Gold nanoparticle (AuNP) encapsulated with a di-block copolymer, polystyrene-block-poly(acrylic acid) (PSPAA), is a type of core-shell nanoparticle (AuNP@PSPAA). This structure composes of a metallic core and an organic amphiphilic polymer, which serves two important functions. Firstly, the hydrophobic polystyrene (PS) block of the polymer is able to self-assemble around the thiol-functionalized AuNP, protecting the core. Secondly, the poly(acrylic acid) (PAA) block helps maintain charge-charge repulsion, and hence allows the AuNP@PSPAA to maintain its stability in water [6]. While pores or voids may be found in the PS domain when placed in water [8], AuNP@PSPAAwere found to resist cyanide etching for months [9]. This suggests that limited or low ionic diffusion of ions occurred across the polymer shell.
In the literature, various diffusion models in polymers have been proposed [4]. These models are usually based on the free volume theory, which is derived from Fick's law of diffusion [10], where small holes residing in the polymer will form the basis of the diffusion of molecules across the polymer [11]. For example, Cohen and Turnbull developed a model where the diffusion coefficients of the molecules will decrease exponentially as its size increases, and also provided a mean to understand the probability of finding holes in a polymer [12]. Fujita extended Cohen and Turnbull work's and derived a new model, which could explain the diffusion of molecules in a solvent-polymer system [13]. This model has also been adopted to study different types of polymer including poly(methyl methacrylate) and poly $(\mathrm{N}, \mathrm{N}-$ diethylacrylamide) $[4,14]$. Collectively, these works highlight the importance of understanding diffusion in polymer.

${ }^{*}$ Corresponding author: Zairong Phua, Department of Chemistry and Biological Chemistry, Nanyang Technological University, Singapore, Tel: 12173778768; E-mail:1430519741@qq.com

Received: October 06, 2017; Accepted: October 11, 2017; Published: October 17, 2017

Citation: Song X, Phua Z, Xu W, Liu (2017) Probing lonic Diffusion Dynamics through the Modulation of Polymer Shell Using Surface-Enhanced Raman Spectroscopy. J Nanomed Nanotechnol 8: 465. doi: 10.4172/2157-7439.1000465

Copyright: $\odot 2017$ Song $X$, et al. This is an open-access article distributed under the terms of the Creative Commons Attribution License, which permits unrestricted use, distribution, and reproduction in any medium, provided the original author and source are credited. 


$$
D=A R T \exp \left(-\frac{B}{f_{v}}\right)
$$

where $D$ is self-diffusion coefficient of the molecule, $A$ is a constant, $R$ is the real gas constant, $T$ is temperature, $B$ is the particle size, $f_{v}$ is the average free volume per molecule.

Several studies involving core-shell nanoparticles have revealed their utility in terms of diffusion of either small molecules or ions, by relating to some diffusion models. For example, $\mathrm{AuCl}_{4}^{-}$was able to diffuse across the polymer shell of Au@Oligoaniline nanoparticle at a very slow rate [7]. Presence of small amounts of organic solvent can swell polymeric structures, promoting penetration of ions [15]. Polymers, conductive ones, may be doped using acids or bases to support ionic conductivity [5]. However, it is not easy to fully comprehend how ionic diffusion will occur at the nanoscale. Therefore, it is necessary to find an easy and simple method to probe this process.

In this study, the ionic diffusion of cyanide through the polymer shell of AuNP@PSPAA was probed using surface enhancement Raman spectroscopy (SERS) [16]. We envision that as cyanide etches the AuNP, its surface ligands will be detached, contributing to a decrease in SERS signal. We hypothesize that when the PS domain of the polymer becomes more porous, more water will be able to penetrate through these pores, serving as a medium to support the cyanide diffusion to etch the AuNPs. This provides a useful platform to probe the effect of ionic diffusion through the predominately hydrophobic of the PS membrane.

To better understand the ionic diffusion of cyanide, the porosity of the PS was modulated using various conditions, allowing the entry of ions across the hydrophobic membrane to be carried out at differently. The four different approaches were adopted in our study: (a) swelling the PS domain using an organic solvent of varying amounts; (b) incubating the AuNP@PSPAA for a long period of time in water; (c) incorporating aniline and its oligoaniline into the PSPAA shell to increase its polarity; and (d) using cholesterol as a biomolecule to induce defect formation.

\section{Results and Discussion}

Raman active ligands such as 2-napthalenthiol (2-NT) can produce characteristics SERS spectrum when attached onto metallic surfaces [17]. In this study, this ligand was chosen to tune the interfacial energy between Au-polymer [18], forming core-shell nanoparticles of AuNP@ PSPAA [19]. In order to probe the effect of ionic diffusion, small amount of cyanide was added and allowed to be diffused across PS, under varying experimental parameters, to etch the AuNPs over 90 h. We monitored the Raman shift of $1067 \mathrm{~cm}^{-1}$ corresponding to the symmetric C-H bending vibrational mode of $2 \mathrm{NT}$, which has a strong SERS intensity [17], and envisioned that as ionic diffusion of cyanide proceeds, the intensity of this band will decrease. As the AuNPs are being etched by the cyanide ions, soluble ions of $\mathrm{Au}(\mathrm{CN}) 2$ - will be formed. This ion was also found to be SERS active and will produce a Raman signal, of a high frequency mode, at $\sim 2100 \mathrm{~cm}^{-1}$ [20]. Thus on this basis, we were able to monitor the ionic diffusion of cyanide in situ.

\section{AuNP@PSPAA in THF}

Swelling of a polymer is an important process because it allows the polymer to be converted from a glassy to rubbery state. For example, many organic solvents such as DMF, dioxane, acetone, benzene and tetrahydrofuran (THF) can cause polystyrene to swell. When the organic solvent penetrates into the polymeric network, they are initially inserted into free holes or voids of the polymer. Next, as more solvent diffuses into the network, the solvent molecules will form an infiltration layer and cause the polymer to be completely swollen over time. When large excess of the solvent eventually infiltrate the polymer, dissolution of the polymer will be expected, forming a solute-solvent solution [21].

Ando et al. demonstrated that poly (N,N-diethulacrylamide) may be swollen by using deuterated water and poly(ethylene glycol) (PEG). The swollen state of the polymer was probed using proton $(1 \mathrm{H})$ nuclear magnetic resonance (NMR) and found that the diffusion of the water can be explained by the free volume theory [14]. Similarly, Taton et al. discovered that when small amount of THF was used to swell the cross-linked block-copolymer, ionic diffusion of cyanide resulted in immediate etching of the embedded AuNP in the polymer [15]. These studies illustrate probing the swollen state of the polymer is of great importance in understanding the transport of molecules (Figures 1 and 2).

\section{a AuNP@PSPAA - THF/Water}

\section{b $\quad 1067 \mathrm{~cm}^{-1}$ Band}
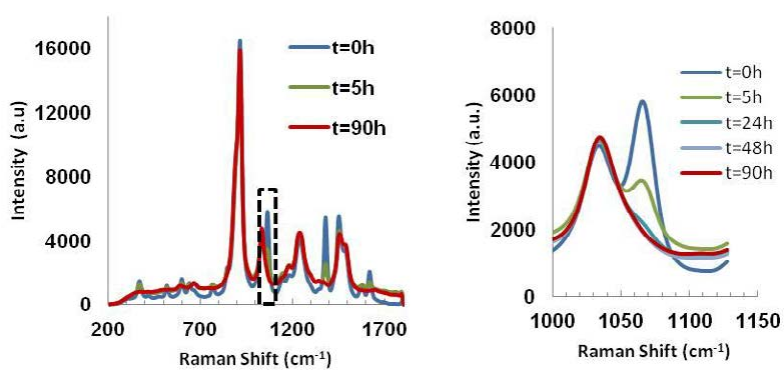

C

Etching of AuNP@PSPAA@THF/Water

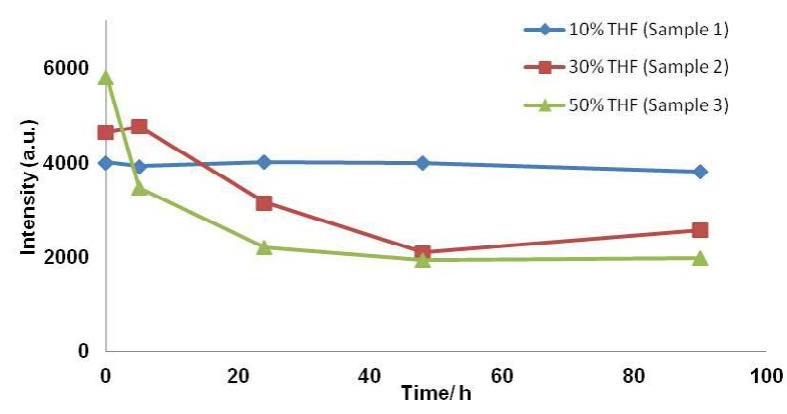

Figure 1: Raman spectra illustrating the changes of SERS intensity vs. time when AuNP@PSPAA are etched by cyanide with the presence of THF (a)THF/ Water (1:1); (b) magnified SERS band of (a) at $1067 \mathrm{~cm}^{-1}$; (c) with increasing amount of THF (10,30 and 50\% - Sample 1, 2 and 3 respectively) monitored over $90 \mathrm{~h}$.
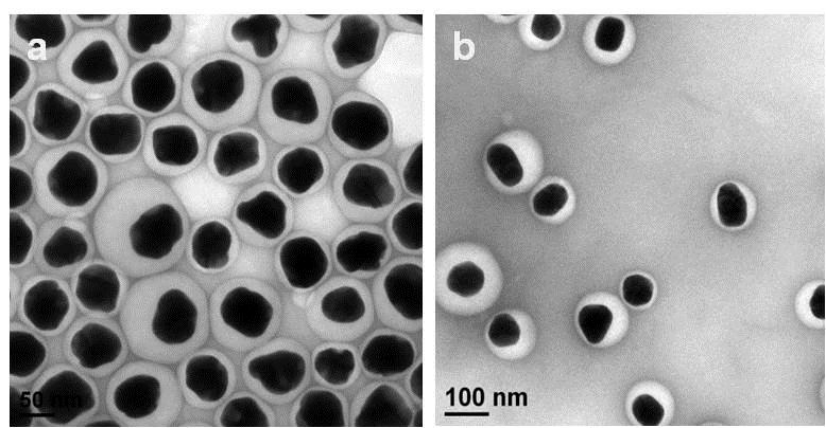

Figure 2: TEM images showing the purified AuNP@PSPAA were incubated in different solutions: (a) after $24 \mathrm{~h}$ in presence of cyanide in $30 \%$ THF solution; (b) $90 \mathrm{~h}$ in the absence of cyanide in $50 \%$ THF solution. 
In our study, we chose THF as the organic solvent to swell PS for three reasons. Firstly, cyanide cannot react with THF and will not interfere with the etching process. Secondly, the low dielectric constant of THF will make it distinct to us that during the process of etching, water served as the medium in which the cyanide ions will diffuse on. Lastly, THF and water can form a miscible liquid mixture with AuNP@ PSPAA.

We swelled the PS domain by using an increasing amount of THF: 10,30 and $50 \%$ (Sample 1, 2 and 3 respectively) and monitored the rate of etching over several data points for $90 \mathrm{~h}$ (Figure 2c). It is found that the colour of all three samples did not visibly change when cyanide was added initially. In contrast, bare citrate-stabilized AuNPs are able to be etched immediately, resulting in a visible change in colour from red to colourless within seconds. This result suggests that the ions needed time, in order to diffuse through the hydrophobic domain even in the presence of THF. After $5 \mathrm{~h}$, we discovered that slight visible colour change could be seen in the sample 3 only. The SERS intensity of this sample dramatically decreased at $1067 \mathrm{~cm}^{-1}$ Raman band (Figure $2 \mathrm{~b}$ ). As the samples were incubated in the presence of cyanide for $24 \mathrm{~h}$, more visible etching and decrease in SERS intensity was recorded in Sample 2 and 3. Increasing the time to $48 \mathrm{~h}$ and $90 \mathrm{~h}$, the SERS intensity appeared to be stabilized. Control experiment shows that the SERS intensity slightly decreased in the absence of THF, even after $90 \mathrm{~h}$. The results indicate that THF molecule can insert in the polymer chains and swell the polymer shell. As a result, the porosity of polymer shell increased, allowing the diffusion of water and cyanide. As shown in Figure $2 c$, the diffusion rate of cyanide increased with increasing the amount of THF. Interestingly, low THF content (10\%, sample 1$)$ has no effect on the ionic diffusion over $90 \mathrm{~h}$ (Figure $2 \mathrm{c}$ ). We postulated that the small amount of THF may not be able to swell the polymer sufficiently. Instead, the THF could have been only resided in the holes or voids that are found within the PS domains. The low mobility and fluidity of PS chains may be not sufficient to cause water to penetrate deeper to etch the embedded AuNPs.

In our experiments, polymer shell of AuNP@PSPAA is in an uncross-linked state. This might lead one to contemplate that the large amount of solvent might cause complete dissolution of the polymer shell surrounding the AuNP. Figure $3 \mathrm{~b}$ shows the result when the

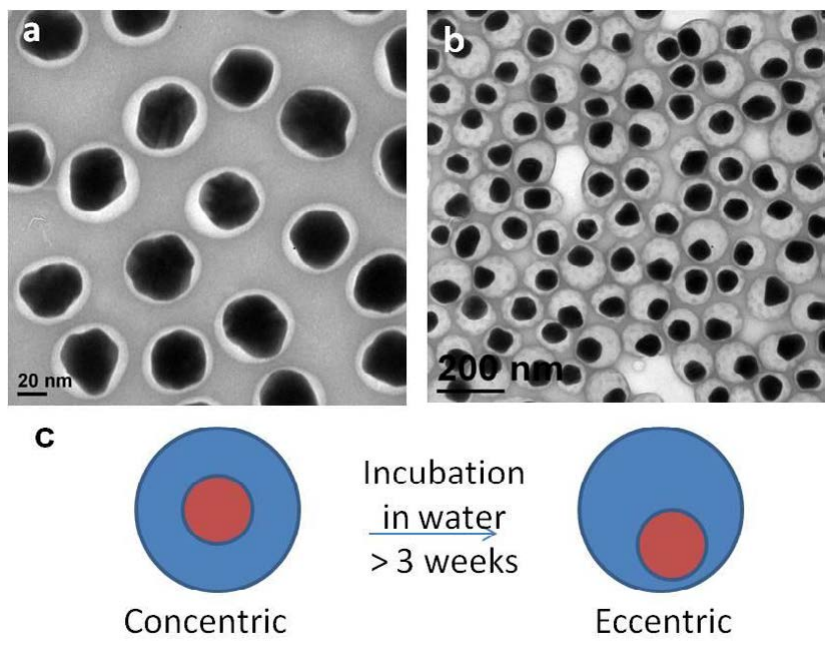

Figure 3: TEM images showing purified AuNP@PSPAA (a) before incubation (b) incubated in water for more than three weeks; (c) Core-shell NP turned eccentric.
AuNP@PSPAA were incubated in 50\% THF solution for $90 \mathrm{~h}$. It is clear that the polymer shell is still intact, which is evidence that the THF is well inserted among the PS chains without compromising the structure of the polymer shell. Moreover, as shown in Figure 3a, the surface of the AuNPs appeared to have been etched away by cyanide when the AuNP@PSPAA were incubated in 30\% THF solution for $24 \mathrm{~h}$. We also observed that the etching of the AuNPs surface appeared to be nonuniformed in nature. This indicates that it is hard to precisely control the entry of the cyanide ions as the solvent molecules are inserted between the polymer chains in a random manner, which results in non-uniformed etching of the AuNPs surfaces.

Collectively, our data is consistent with diffusion of solvent in the polymer $[21,22]$. While we understand that increasing THF can swell the polymer shell leading to a larger increase in volume, it is difficult to characterize the nature of the free volume in the polymer as the PS chains are free to move about in the presence of the solvent. Therefore, the application of the free volume theory is not expected.

\section{AuNP@PSPAA Incubation in water}

It is believed that small cavities could be found between the PS chains [8]. Therefore, it is likely that as time proceeds, water is able to penetrate through the cavities of the PS chains and eventually allow more pores to be formed [23]. We investigated this effect by conducting two experiments. Firstly, the purified as-synthesized AuNP@PSPAA (Figure 4a) were etched in the water and monitored over $90 \mathrm{~h}$. In the second experiment, the NPs were incubated in water for a long period of time (i.e more than 3 weeks), on a shaker, before etching. The rationale of conducting these two experiments is to allow us to control the ability of water to swell the polymer; similar to that of using organic solvents. We predict that the longer the time the NPs are incubated, more water molecules are able to be trapped inside the PS chains, allowing the polymer to swell, which in turn increases it porosity.

Figure $4 \mathrm{~b}$ shows that the concentric AuNP@PSPAA became eccentric after incubation in water over a long time. Most interestingly, dark spots were observed on the polymer shells. It reveals new pores or cavities were formed among the PS domains, so that negative stain was able to diffuse in and enhanced the contrast between PS and background. We postulate that two reasons could result in this phenomenon: Firstly, when the NPs are incubated for a long period, the water molecules are able to diffuse through the cavities residing in the outer layer of the PS chains [23] and subsequently diffuse inwards to the inner polymer shell. It is expected that as water molecules diffuse inward, the mobility of the PS chains will increase even when it is below its glass transition temperature [21]. When the water content in the cavities exceeds its maximum holding capacity, the polymer shell is forced to crystallize by forming aggregates with adjacent PS chains, resulting in the formation of new pores or cavities [24]. Secondly, when incubated in water, the polymer shell and the PS chains will reorganize and crystallize, which decreased the interfacial energy of the polymer shell, and forced the AuNP to become eccentric [18].

A very slow decrease in count rate was observed when the NPs were etched immediately (Figure $5 \mathrm{c}$ ), suggesting that very low ionic diffusion of cyanide occurred across PS. The result is consistent with what we expected because water, unlike organic solvents, cannot swell PS effectively. Instead, water can only penetrate across the polymer shell by first utilizing small voids or pores that are found in PS and slowly making its way through it. In the process, water may help to increase the mobility of adjacent PS chains to help increase the uptake of water. This also helps to support the ionic diffusion of cyanide to etch 


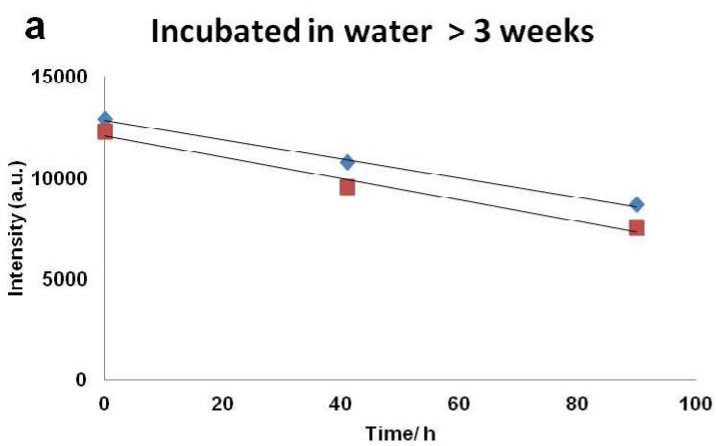

C AuNP@PSPAA etching w/o incubation

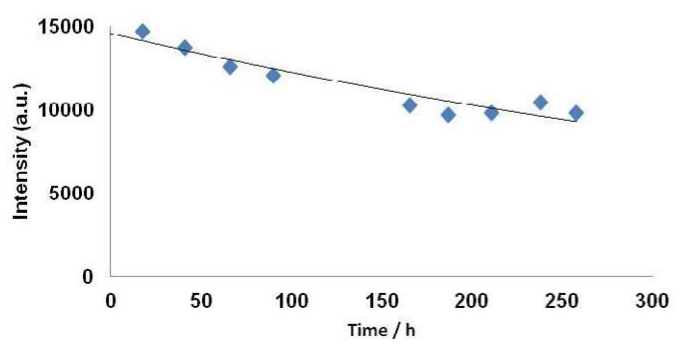

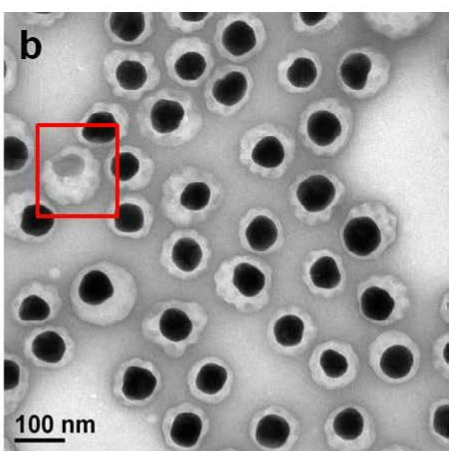

d

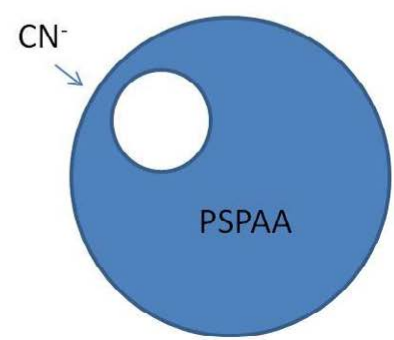

Figure 4: (a) Decrease in SERS intensity of AuNP@PSPAA incubated in water for 3 weeks when etched by cyanide ions; (b) TEM image of the etched AuNPs; (c) Decrease in SERS intensity of AuNP@PSPAA without incubation and monitored for over $250 \mathrm{~h}$; (d) Schematic showing the probable entry of cyanide ions.

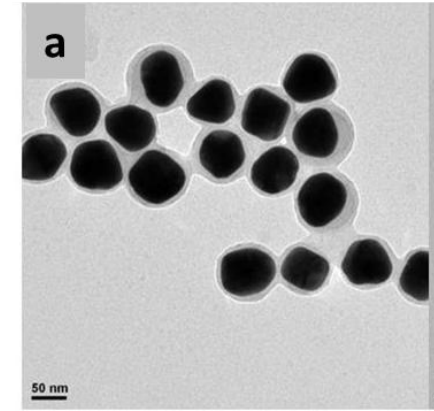

C UV-visible Spectrum
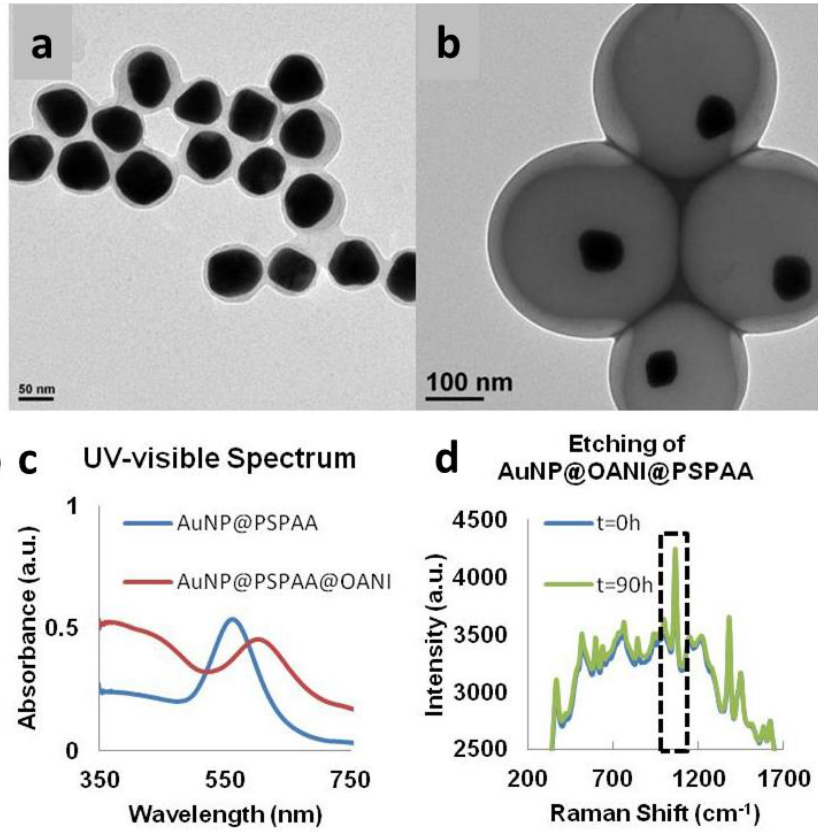

Figure 5: TEM image of (a) AuNP@PSPAA@Aniline; (b) AuNP@PSPAA@ OANI formed using $30 \mu \mathrm{l}$ of aniline; (c) UV-Vis absorption spectra of AuNP@ PSPAA and AuNP@PSPAA@OANI; (d) Etching of AuNP@PSPAA@OANI.

the embedded AuNPs. On inspection of the resultant TEM image after $90 \mathrm{~h}$, we found that essentially all the AuNPs retained their size. At the same time, there was no observable change in colour in the solution. On the basis of the slow decrease in count rate, we postulate that only a very thin layer of AuNP, on its surface, was being etched away, which cannot be clearly observed via TEM characterization. Even over a longer period of time $(250 \mathrm{~h})$, the SERS intensity still remained very strong. In contrast, a more observable decrease in count rate was seen when the AuNP@PSPAA were subjected to incubating in water for a long time (>3 weeks) (Figure 5a). After etching, the colour of the solution turned from red to pale red. Figure 5b shows the TEM image of AuNP@ PSPAA after etching, most of the AuNPs remained unetched, with no observable decrease in sizes. Importantly, empty PSPAA polymer shell was observed in Figure 5b, indicating the AuNP was completely etched by cyanide ions. This may be due to two possible reasons: first, after incubation in water for long time, concentric AuNP@PSPAA evolved into Janus nanoparticles, thus, the exposed AuNPs were easily etched by cyanide ions. Second, concentric NPs changed into eccentric ones, the AuNPs of which are still protected by polymer shells. We postulate that larger defect may form in thinner side of polymer shell, due to reorganization of the polymer shell after incubated in water for long time. As a result, it promotes the entry of the cyanide ions and etching of AuNPs. Although long period of incubation in water appeared to have increased the porosity of the polymer shell, water is still not able to swell the polymer shell sufficiently to increase the rate of ionic diffusion.

Our results suggest that water is able to penetrate through the cavities of the PS chains and allow ionic diffusion of cyanide across the PS domains. However, the reproducibility of the above experiment may be low as it is dependent on the two factors: A longer incubation period might allow more water to swell the polymer shell, while an increase in shaking rate allow water to penetrate deeper into the PS domains and reorganize the shell. Therefore, an increase in both factors may eventually result in more AuNPs being etched away when cyanide is added.

To better understand this effect, a control experiment was set up, where the core-shell NPs were incubated in a DMF/Water $(\mathrm{V} / \mathrm{V}=4: 1)$ 
solution for the same period of time. It was revealed that no visible changes were observed on the polymer shell after purification. This suggests that as DMF is well inserted between the polymer shell, and therefore localized crystallization will not be observed as the organic solvent is capable of keeping the PS chains mobile. TEM analysis of the NPs resembled that of a typical as-synthesized AuNP@PSPAA, suggesting that there could be no visible changes in the polymer shell.

\section{AuNP@Aniline@PSPAA@ and AuNP@Oligoaniline@ PSPAA}

AuNP encapsulated by oligoanline (OANI) was found to support the diffusion of $\mathrm{AuCl}_{4}$ - across its polymer shell [7]. On the basis of this, we attempted to incorporate aniline into the polymer shell of AuNP@ PSPAA to increase both the polarity and porosity of PS. Owning to the polarity of the molecule, we postulate that they would be able to trap water molecules and thereby support ionic diffusion of cyanide through the polymer shell of AuNP@PSPAA.

AuNP@PSPAA@Aniline was prepared using the as-synthesized AuNP@PSPAA. $400 \mu \mathrm{L}$ AuNP@PSPAA was purified and dispersed into $1 \mathrm{~mL}$ of $\mathrm{NaOH}(0.1 \mathrm{mM})$ solution containing $10 \mu \mathrm{L}$ of aniline. The resultant solution was stirred at $500 \mathrm{rpm}$ maintained at $75^{\circ} \mathrm{C}$. As shown in Figure 6a, the resultant core-shell AuNP@PSPAA@ Aniline revealed a double-layered polymer shell. It is believed that the hydrophobic aniline diffused into PSPAA shells to reduce the anilinewater interfacial tension [18]. Heating promotes the mobility of the PS chains and stirring increases the diffusion of small molecules, thus, the diffusion of aniline enhanced under these conditions. When the resultant solution is being cooled to room temperature, the mobility of the PS chain will be greatly reduced and this will allow aniline to be trapped inside PS domain [25]. This new core-shell structure is believed to be composed of a hard and soft-shell. The soft-shell being the aniline layer whilst the hard-shell being the PS. The aniline layer is termed as a soft-shell because the molecules behave very liquid-like at room temperature. On the other hand, the PS chains are very rigid at room temperature and are termed as a hard-shell.

Our etching experiment involving AuNP@Aniline@PSPAA revealed that no observable changes in the SERS intensities; it indicates that cyanide might not diffuse through the hard and soft polymer shell. This result may be explained by various factors: Firstly, with presence of the hard and soft shell, it is very difficult for cyanide to diffuse through the PS (hard-shell) and hence it might not reach the soft-shell to etch the embedded AuNP. Secondly, even if cyanide might have reached the soft-shell, the low conductivity of aniline [26] coupled with its low dielectric constant [27] will also make diffusion of cyanide difficult.

In order to promote the ionic diffusion of cyanide, we modified our experiment by adding a small amount of oxidant to induce the

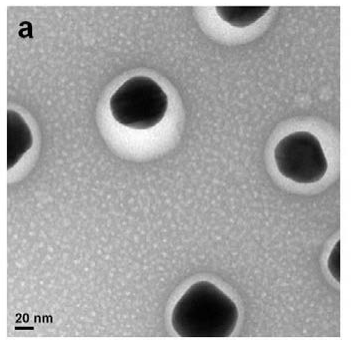

\section{b Raman Spectrum of} AuNP@Cholesterol@PSPAA

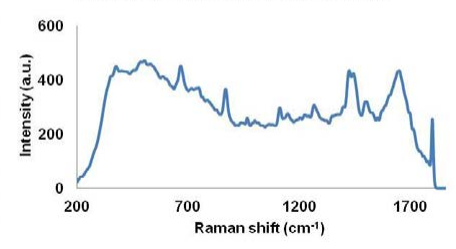

Figure 6: (a) TEM image of AuNP@Thiocholestrol@PSPAA; (b) Raman spectrum. formation of OANI, which allowed us to increase both the porosity and polarity of the polymer shell. Immediately after the synthesis of AuNP@ PSPAA@Aniline, $10 \mu \mathrm{L}$ of $\mathrm{H}_{2} \mathrm{O}_{2}(10 \mathrm{mM})$ and $5 \mu \mathrm{L}$ of $\mathrm{FeCl}_{3}$ were added and stirred, with the temperature maintained at $85^{\circ} \mathrm{C}$ for $2 \mathrm{~h}$. The resultant NP will look very similar to that as shown in Figure 6b. On the other hand, Figure $6 c$ shows the TEM image of AuNP@PSPAA@OANI made using thrice the amount of reagents, it is clear that a doublelayered shells will be formed. By increasing the concentration of the reagents (Aniline, $\mathrm{H}_{2} \mathrm{O}_{2}$ and $\mathrm{FeCl}_{3}$ ) by three times, we observed that the size of the polymer shell increased significantly. This suggests that as we increase the amount of aniline/OANI, more of these molecules are loaded inside the polymer shell, making the core-shell NPs larger. After polymerization, the size of polymer shells dramatically increased compared to that of AuNP@PSPAA@Aniline (Figure 6b).

IR characterization of the compound revealed a broad absorption at around $3300 \mathrm{~cm}^{-1}$ which is likely to be composed of overlapping N-H of the primary amines and $\mathrm{O}-\mathrm{H}$ of water stretches;and also around 1630 $\mathrm{cm}^{-1}$ which could be due to $\mathrm{C}=\mathrm{C}$ and $\mathrm{N}-\mathrm{H}$ bending stretches (Figure 7).UV-vis of AuNP@PSPAA@OANI showed a red-shift phenomenon with an absorbance at around $590 \mathrm{~nm}$ (Figure 6d). We think that the change in UV-vis absorbance could be due to the change in reflective index of the resultant polymer shell and the size of polymer shell [28]. While complete characterization of the state of polymerization of aniline was not carried out, it is unclear if any oilgomers were formed. We postulate that they could be dimer or trimers, of very small amounts, owning to the absence of characteristics IR peaks between $\sim 690$ and $750 \mathrm{~cm}^{-1}$ of monosubstitured aromatic rings $[29,30]$. Additionally, the presence of the hydrogen peroxide as oxidant could also help to support the formation of nitrosobenzene [31]. Therefore, it is believed that a range of compounds derived from aniline could be present in the soft-shell. However, in our discussion the resultant NP is termed as AuNP@OANI@PSPAA for convenience.

WhenAuNP@PSPAA@OANI was etched by cyanide, no observable decrease in SERS intensities was recorded (Figure 6e). Strangely, even with the incorporation of both aniline and its oligomers, ionic diffusion of cyanide was not observed. This leads us to think that the doublelayered shell has low porosity and is limited to penetration of water or ions. Also, as discussed earlier, it could also be due to the presence of the hard-shell PS blocks that limits the diffusion of ions

\section{AuNP@PSPAA@Cholesterol}

In the biological system, a cellular membrane acts as a barrier to help control the diffusion of small molecules or ions in and out of the cell. The decrease in permeability of molecules and ions has been linked to the present of cholesterol in the cellular membrane as it allows the phosolipid bilayer to become sufficiently more hydrophobic, preventing the entry of water [32,33]. Traditionally, the study of the permeability of cholesterol in the membrane involves the measurement of glucose release from lecithin vesicles. In order to help them understand the permeability effect, both glucose dialysis and nuclear magnetic resonance methods were adopted in the study by the authors [34]. This method, however, may be tedious owning to the scale of preparation and quantification. For this reason, we decided to incorporate this molecule into our system - to test its ionic permeability on the PS domain - because AuNP@PSPAA resembles very much like a biological cell, which is a simpler approach. While many methods such as loading or during PSPAA assembly can be adopted to incorporate cholesterol into the PS [35], these approaches may not suitable as its relative position with respect to PSPAA cannot be well controlled or established. Hence, we used a cholesterol ligand which contains a thiol 
a<smiles>Sc1ccc2ccccc2c1</smiles>

\section{2-napthalenethiol}<smiles>CC(C)CCCC(C)[C@H]1CC[C@H]2C3C=C4CC(S)CCC4(C)C3CC[C@]21C</smiles>

Thiolcholesterol

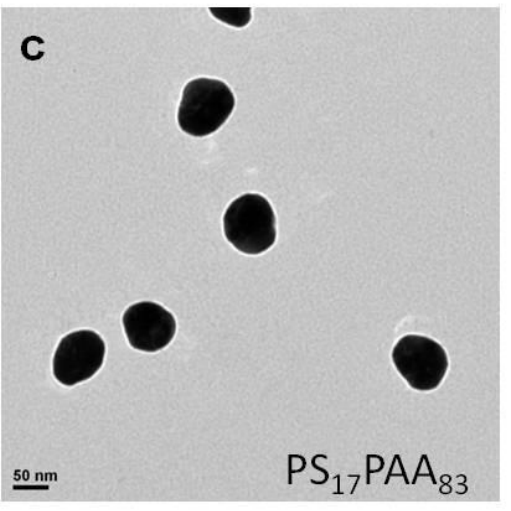

b Evidence of Dual - Ligands

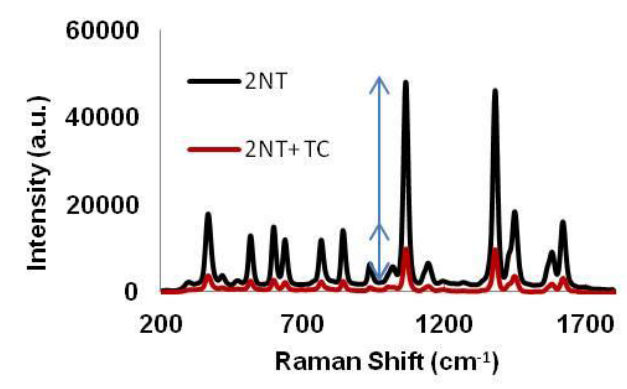

d

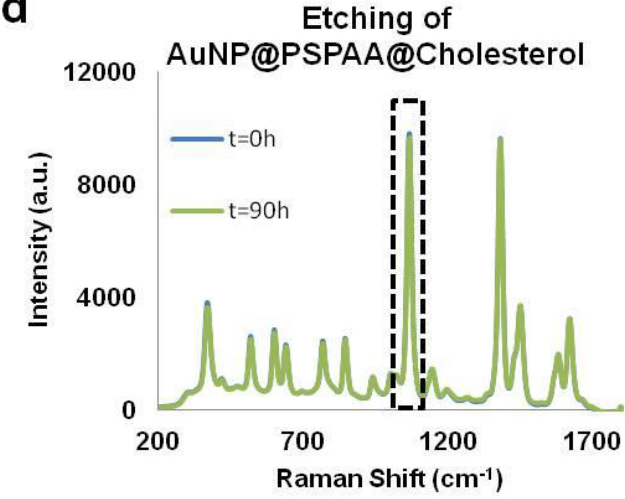

Figure 7: (a) Structures of the ligands: 2-napthalenethiol (2NT) and thiolcholesterol (TC), respectively; (b) SERS spectra of dual-ligands; (c) TEM image of AuNP@ PSPAA using dual-ligands as surface ligands; (d) SERS spectra showing the etching of AuNP@PSPAA, when cholesterol was used as surface ligand.

group to functionalize the AuNP, enabling us to position the ligand properly with PSPAA.

Complete encapsulation of PSPAA using thiocholesterol (TC) can be observed, which clearly suggests that the ligand is capable of attaching onto the AuNP surface. In addition, this result also indicates that cholesterol and polystyrene form stable interface [36], forming the core-shell NP. While the resulting AuNP@Cholesterol@PSPAA allows us to probe the effects of cholesterol in relation to its ionic permeability with PS, the lack of strong SERS signal makes it difficult for us to monitor the rate of cyanide diffusion. Therefore, we adopted a dual ligand approach, by using both $2 \mathrm{NT}$ and $\mathrm{TC}$, where we are able to make use of the SERS signals of the former ligand [17] to probe the effects of ionic diffusion across PS. Additionally, we also chose a shorter PSPAA chain, consisting of 17 units of PS and 83 PAA, to better understand the cholesterol-PS interaction.

The successful attachment of the dual ligands may be rationalized by observing the difference in SERS intensity. The combination of $2 \mathrm{NT}$ and TC produced a much lower SERS intensity as compared to as when only $2 \mathrm{NT}$ is used. Presently, we can rule out the fact that isotropic attachment of each of the ligands as no nanocavity was observed under TEM [9]. Hence, it is likely that the dual ligands are able to form mixed anisotropic monolayers on the surface of the AuNPs since both ligands are hydrophobic in nature. However, one drawback of using this technique is that owning to the instability of using a short PSPAA co-block polymer, partial aggregation might be observed. Our etching result suggests that little or no ionic diffusion of cyanide occurred during our observation period. Control experiment using $2 \mathrm{NT}$ as ligand and PS17PAA83 produced similar result, implying that cholesterol did not play a significant role in modulating the function of PS. While cholesterol is capable of reducing intrinsic defects in the cellular membrane and thereby reducing permeability of water,3 we feel that larger defects [37] should be apparent in the cholesterolPS interface owning to steric repulsion of the methyl group on the cholesterol molecule, which should result in faster ionic diffusion of cyanide. However, it is not easy for us to probe the nature of the pores or the hydrophobic pockets that are formed in the cholesterol-PS interface. Another reason could be because the resulting cholesterolPS interface is too rigid [38] and it is not fluid enough to cause the movement of the PS chains to induce the entry of water into the hydrophobic membrane. Thus, this resulted in no observable etching or ionic diffusion of cyanide.

\section{Etching rates and diffusion coefficients of cyanide}

From our series of experiments, we found out that the ionic diffusion of cyanide through PS appeared to be fastest when swelled with an organic solvent and followed by incubation in water. Also, cyanide appeared to be impenetrable when we attempted to modulate PS using cholesterol, aniline and its oligomers. In the literature, the diffusion coefficient of cyanide is $2.07 \times 10^{5} \mathrm{~cm}^{2} \mathrm{~s}^{-1}$ [39]. Ideally, from this value we may be able to appreciate how fast or slow the diffusion of cyanide is through PS domain by observing the change in SERS intensity over time. However, it remains difficult to estimate or calculate the diffusion coefficient of the cyanide ions as more than one factor needs to be accounted for. Firstly, when PS is swelled by the co-solvent or water, it is difficult to characterize the nature of the free volume formed. Moreover, the PS chains may become slightly mobile in nature and allows the cyanide ions to jump from one void to the other making it even harder to understand its mode of diffusion. Secondly, for one to work out the diffusion coefficient of the ions, the diffusion process has to be the rate-determining step [40]. In our system, in order for the AuNP to be etched, we have to consider the 
Citation: Song X, Phua Z, Xu W, Liu (2017) Probing Ionic Diffusion Dynamics through the Modulation of Polymer Shell Using Surface-Enhanced Raman Spectroscopy. J Nanomed Nanotechnol 8: 465. doi: 10.4172/2157-7439.1000465

Page 7 of 8

inward diffusion of both the cyanide ions and the oxygen molecules [7]. Thirdly, it remains very hard for us to quantify the SERS intensities as currently no tool or model is available to us for means of calculation [41]. The SERS intensities will depend several factors such as particle sizes, Raman cross section [42] and concentration of the AuNPs [43], which will make it too complex for us to control [44,45].

\section{Conclusion}

In this study, we modulate the porosity of the polymer shell of AuNP@PSPAA by using several conditions such as organic solvent, incubation in water, using aniline to increase its porosity and cholesterol to induce defect formation. We found that as we increase the porosity of the polymer shell, ionic diffusion of cyanide across PS can increase significantly and most notably when using the organic solvent (e.g., THF). This effect was monitored in situ by using SERS and it has proved to be a very simple and effective tool to probe this effect. While SERS provides us with a means to probe ionic diffusion of cyanide, specific diffusion coefficient of ions was not discussed in our study as we are not able to develop a single quantification tool. Future work will be focused on using the same technique to increase our mechanistic understanding of ionic diffusion in polymer by using conductive polymers such as polyaniline.

\section{References}

1. Smitha B, Sridhar S, Khan AA (2005) Solid polymer electrolyte membranes for fuel cell applications - a review. Journal of Membrane Science 259: 10.

2. Tanak Y, Yoshinobu T (2007) Membrane Science and Technology Ed. Elsevier 12: 37 .

3. Yeagle PL (1991) Modulation of membrane function by cholesterol. Biochimie 73: 1303.

4. Masaro L, Zhu XX (1999) Physical Models of Diffusion for Polymer Solutions Gels and Solids. Progress in Polymer Science 24: 731

5. Virji S, Huang J, Kaner RB, Weiller BH (2004) Polyaniline Nanofiber Gas Sensors: Examination of Response Mechanisms. Nano Letters 4: 491-496.

6. Wang H, Song XH, Liu C, He J, Chong W, et al. (2014) Homo- and Copolymerization of Polysytrene-block-Poly(acrylic acid)-Coated Metal Nanoparticles. ACSNano 8: 8063-8073.

7. Sun H, Shen X, Yao L, Xing S, Wang H, et al. (2012) Measuring the Unusually Slow lonic Diffusion in Polyaniline via Study of Yolk-Shell Nanostructures. Journal of the American Chemical Society 134: 11243-11250.

8. Gowd EB, Tashiro K, Ramesh C (2009) Structural phase transitions of syndiotactic polystyrene. Progress in Polymer Science 34: 280

9. Tan, LH, Xing S, Chen T, Chen G, Huang X, et al. (2009) Fabrication of Polymer Nanocavities with Tailored Openings. ACS Nano 3: 3469.

10. Lehner FK (1979) On the validity of Fick's law for transient diffusion through a porous medium. Chemical Engineering Science 34: 821.

11. Vrentas JS, Vrentas CM (2003) Evaluation of the free-volume theory of diffusion. Journal of Polymer Science Part B: Polymer Physics 41: 501-507.

12. Cohen MH, Turnbull D (1959) Molecular Transport in Liquids and Glasses. The Journal of Chemical Physics 31: 1164

13. Fujita H (1961) In Fortschritte Der Hochpolymeren-Forschung. Springer Berlin Heidelberg.

14. Matsukawa S, Ando I (1997) A Study of Self-Diffusion of Molecules in Polymer Gel by Pulsed-Gradient Spin-Echo 1H NMR. Macromolecules 29: 7136-7140.

15. Kang Y, Taton TA (2005) Core/shell gold nanoparticles by self-assembly and crosslinking of micellar, block-copolymer shells. Angewandte Chemie International Edition 44: 409-412.

16. McQuillan AJ (2009) The discovery of surface-enhanced Raman scattering. Notes and Records of the Royal Society 63: 105

17. Alvarez-Puebla RA, Dos Santos DS, Aroca RF (2004) Surface-enhanced Raman scattering for ultrasensitive chemical analysis of 1 and 2-naphthalenethiols. Analyst 129: 1251-1256

18. Torza S, Mason SG (1970) Three-phase interactions in shear and electrical fields. Journal of Colloid and Interface Science 33: 67.

19. Chen T, Wang H, Chen G, Wang Y, Feng Y, et al. (2010) Hotspot-Induced Transformation of Surface-Enhanced Raman Scattering Fingerprints. ACS Nano 4: 3087-3094.

20. Beltramo GL, Shubina TE, Mitchell SJ, Koper MTM (2004) Cyanide adsorption on gold electrodes: a combined surface enhanced Raman spectroscopy and density functional theory study. Journal of Electroanalytical Chemistry 563: 111

21. Miller-Chou BA, Koenig JL (2003) A review of polymer dissolution. Progress in Polymer Science 28: 1223.

22. Jones RC, Gainer JL (1976) Diffusion in Mixed Polymer Solutions. Industrial \& Engineering Chemistry Fundamentals 15: 83-85.

23. O'Kane JM, Sherrington DC (1990) Hysteresis-like behavior in the swelling/ deswelling of polystyrene crosslinked resins using binary solvent mixtures Macromolecules 23: 5286

24. Aref-Azar A, Arnoux F, Biddlestone F, Hay JN (1996) Physical ageing in amorphous and crystalline polymers. Thermochimica Acta 273: 217.

25. Blinova NV, Reynaud S, Roby F, Trchová M, Stejskal J (2010) The polymerization of aniline in polystyrene latex particles. Synthetic Metals 160 1598-1602.

26. Pound JR (1927) The Electrical Conductivities of Some Solutions in Aniline. The Journal of Physical Chemistry 31: 547-563.

27. Wohlfarth C (2008) In Supplement to IV/6; Lechner MD (ed.) Springer Berlin Heidelberg 17: 948.

28. Jalbout AF, Jiang T (2002) Ab-Initio molecular geometry and normal coordinate analysis of pyrrolidine molecule. Spectrochimica Acta Part A: Molecular and Biomolecular Spectroscopy 58: 2647-2657.

29. Laska J, Widlarz J (2005) Spectroscopic and structural characterization of low molecular weight fractions of polyaniline. Polymer 46: 1485

30. Feng X, Yang G, Xu Q, Hou W, Zhu JJ (2006) Self-Assembly of Polyaniline/ Au Composites: From Nanotubes to Nanofibers. Macromolecular Rapid Communications 27: 31-36

31. Zhu Z, Espenson JH (1995) Kinetics and Mechanism of Oxidation of Anilines by Hydrogen Peroxide As Catalyzed by Methylrhenium Trioxide. The Journal of Organic Chemistry 60: 1326-1332.

32. Ross PD, Subramanian S (1981) Thermodynamics of protein association reactions: forces contributing to stability. Biochemistry 20: 3096-3102.

33. Yeagle PL (1985) Cholesterol and the cell membrane. Biochimica et Biophysica Acta 822: $267-287$

34. Yeagle PL, Martin RB, Lala AK, Lin HK, Bloch K (1977) Differential effects of cholesterol and lanosterol on artificial membranes. Proceedings of the National Academy of Sciences 74: 4924 .

35. Wang $\mathrm{H}, \mathrm{Xu}$ J, Wang J, Chen T, Wang $Y$, et al. (2010) Probing the Kinetics of Short-Distance Drug Release from Nanocarriers to Nanoacceptors. Angewandte Chemie International Edition 49: 8426.

36. Mudgil P, Dennis GR, Millar TJ (2005) Wetting of Regularly Structured Gold Surfaces. Langmuir 21: 1338.

37. Losic D, Shapter J, Gooding JJ (2005) Mapping of defects in self-assembled monolayers by polymer decoratio. Journal of Solid State Electrochemistry 9 : 512-519.

38. Wilhelm V, Hellmann GP (2000) Micellar reinforcement of polystyrene with grafted rigid-chain polyesters. Polymer 41: 1905.

39. Vanysek P (2012) In Handbook of Chemistry and Physics 93.

40. Cochain B, Pinet O, Richet P (2013) Diffusion of sodium ions driven by charge compensation as the rate-limiting step of internal redox reactions. Journal of Non-Crystalline Solids 365: 23.

41. Amendola V, Meneghetti M, Fiameni S, Polizzi S, Fracasso G, et al. (2011) SERS labels for quantitative assays: application to the quantification of gold nanoparticles uptaken by macrophage cells. Analytical Methods 3: 849 . 
Citation: Song X, Phua Z, Xu W, Liu (2017) Probing lonic Diffusion Dynamics through the Modulation of Polymer Shell Using Surface-Enhanced Raman Spectroscopy. J Nanomed Nanotechnol 8: 465. doi: 10.4172/2157-7439.1000465

Page 8 of 8

42. Abalde-Cela S, Aldeanueva-Potel P, Mateo-Mateo C, Rodríguez-Lorenzo L, Alvarez-Puebla RA, et al. (2010) Surface-enhanced Raman scattering biomedical applications of plasmonic colloidal particles. Journal of The Royal Society Interface 7: 435.

43. Feng Y, Wang Y, Wang H, Chen T, Tay YY, et al. (2012) Engineering "Hot" Nanoparticles for Surface-Enhanced Raman Scattering by Embedding Reporter Molecules in Metal Layers. Small 8: 246.
44. Frens G (1974) Controlled Nucleation for the Regulation of the Particle Size in Monodisperse Gold Suspensions. Nature Physical Science 241: 20

45. Feng Y, Xing S, Xu J, Wang H, Lim JW, et al. (2010) Probing the kinetics of ligand exchange on colloidal gold nanoparticles by surface-enhanced Raman scattering. Dalton Transactions 39: 349 